\title{
Extended field intensity-modulated radiotherapy plus concurrent nedaplatin treatment in cervical cancer
}

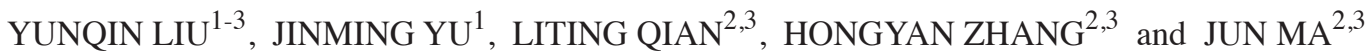 \\ ${ }^{1}$ Department of Radiation Oncology, Shandong Tumor Hospital, Shandong Medical University, Jinan, Shandong 250117; \\ ${ }^{2}$ Department of Radiation Oncology, Anhui Cancer Hospital; ${ }^{3}$ Department of Radiation Oncology, \\ Anhui Provincial Hospital, Anhui Medical University, Hefei, Anhui 230001, P.R. China
}

Received January 20, 2015; Accepted February 26, 2016

DOI: $10.3892 / \mathrm{ol} .2016 .4416$

\begin{abstract}
The present study assessed the efficacy and toxicity of definitive extended-field intensity-modulated radiotherapy (EF-IMRT) plus concurrent chemotherapy in cervical cancer. A total of 48 patients with cervical cancer received the planning target volume between 39.6 and 50.4 Gy in 1.8-2.0 Gy daily fractions, while the enlarged pelvic and/or para-aortic nodes were treated with a total dose of 55-60 Gy in 2.0-2.4 Gy daily fractions using simultaneous integrated boost-IMRT. All patients underwent high dose-rate brachytherapy. Concurrent to EF-IMRT, nedaplatin was administered weekly at a median dose of $30 \mathrm{mg} / \mathrm{m}^{2}$ (range, $25-40 \mathrm{mg} / \mathrm{m}^{2}$ ) for 5 weeks with a total of $150 \mathrm{mg} / \mathrm{m}^{2}$. Of the 48 patients, 46 patients exhibited initial complete responses and 2 patients had partial responses, with a response rate of $100 \%$. After 4-24 months of treatment, 12 patients $(27.08 \%)$ had local and/or distant failure and 39 patients $(81.25 \%)$ were alive at the last follow-up. The 12-month overall survival (OS) and disease-free survival (DFS) were 87.5 and $75.8 \%$, respectively, while the 24-month OS and DFS were 69.7 and $49.7 \%$, respectively. Grade $\geq 3$ acute neutropenia and thrombcytopenia occurred in $20(41.7 \%)$ and $4(8.3 \%)$ patients, respectively, while 2 patients $(4.2 \%)$ developed grade $\geq 3$ diarrhea and $2(4.2 \%)$ had grade $\geq 3$ late toxicities. However, no patients exhibited grade $\geq 3$ vomiting. Thus, concurrent nedaplatin chemotherapy with definitive EF-IMRT was effective and relatively safe for treating patients with cervical cancer. Furthermore, EF-IMRT was able to deliver $\leq 60$ Gy to enlarged para-aortic and/or pelvic nodes using simultaneous integrated boost without increased acute and late gastrointestinal toxicity.
\end{abstract}

Correspondence to: Dr Jinming Yu, Department of Radiation Oncology, Shandong Tumor Hospital, Shandong Medical University, 440 Jiyan Road, Jinan, Shandong 250117, P.R. China

E-mail: yujinmingsd@126.com

Key words: cervical cancer, chemotherapy, extended field, Intensity-modulated radiotherapy, toxicity

\section{Introduction}

Cervical cancer is a significant worldwide health problem, particularly in developing countries. Globally, cervical cancer accounted for the third most commonly diagnosed type of cancer and the fourth leading cause of cancer-related mortality in women in 2008 (1). Patients in the early stages of cervical cancer typically exhibit no symptoms, whereas various symptoms, such as abnormal vaginal bleeding, pelvic pain and pain during sexual intercourse, are observed in the advanced stages $(2,3)$. Cancer screening with the Papanicolaou smear test has been demonstrated to markedly reduce the occurrence of advanced cervical cancer and mortality $(2,3)$. However, treatment of cervical cancer varies significantly; for example, the early stages of the disease are treated with surgery, while the later stages of the disease are treated only with chemotherapy and/or radiotherapy. Generally, cervical cancer is radiosensitive; thus, radiation may be used in all stages of disease, including when surgery is not an option for a patient in the early stages of disease (3). Concurrent cisplatin-based chemoradiotherapy is the standard treatment for locally advanced cervical cancer. Cervical cancer patients with enlarged para-aortic nodes (PAN) may be treated with radical hysterectomy with removal of the lymph nodes or radiation therapy, whereas larger early stage tumors may be treated with radiation therapy and cisplatin-based chemotherapy, hysterectomy with adjuvant radiation therapy, or cisplatin chemotherapy followed by hysterectomy $(3,4)$. By contrast, advanced stages of cervical cancer (stages IIB-IVA) are typically treated with radiation therapy and cisplatin-based chemotherapy $(3,4)$. However, cervical cancer with metastasis to the PAN has a poor prognosis $(5,6)$. In such cases, concurrent cisplatin-based chemotherapy with extended-field radiotherapy (EFRT) is the standard treatment strategy. Previous studies have demonstrated an improvement in disease control using EFRT and concurrent chemotherapy, and have shown a positive effect on overall survival (OS) compared with radiotherapy alone (7-10). However, the high levels of acute and chronic toxicities associated with EFRT are still potential barriers. In addition to decreasing patient quality of life, acute toxicity leads to premature termination of chemotherapy, and the number of chemotherapy cycles is an independent predictor of survival (11). Therefore, there is 
an urgent need to evaluate novel treatment strategies to reduce toxicity and improve treatment efficacy. A previous study discussed the benefits of intensity-modulated radiotherapy (IMRT), which provides more conformal dose distribution into the tumor lesion. Furthermore, IMRT reduces the absorbed dose and the volume of the organ at risk (OAR), thus leading to reduced acute and late toxicity (12). Nedaplatin is an analog of cisplatin that exhibits less nephrotoxicity, neurotoxicity and gastrointestinal (GI) toxicity than cisplatin (13). Thus, the present study assessed the efficacy and toxicity of extended field-IMRT (EF-IMRT) with concurrent nedaplatin treatment in cervical cancer.

\section{Patients and methods}

Ethics. The present study was approved by the Ethics Committee of Anhui Tumor Hospital and Anhui Provincial Hospital (Hefei, China). Informed consent was obtained from all patients included in the current study.

Patients. In the present study, a total of 48 women with histology-confirmed cervical cancer were recruited for treatment with definitive EF-IMRT plus concurrent weekly nedaplatin from Anhui Tumor Hospital and Anhui Provincial Hospital between February 2012 and April 2014. Patients with synchronous malignancies or distant metastases at diagnosis were excluded from the study. Among the total patients, 12 patients exhibited enlarged para-aortic lymph nodes only, 17 patients exhibited multiple enlarged pelvic lymph nodes, and 19 patients had enlarged para-aortic and pelvic lymph nodes. The decision to use EF-IMRT was made by the medical oncologists. Lymph nodes that measured as $>1 \mathrm{~cm}$ in the short axis diameter were considered malignant. Patients with confirmed tumor metastasis to the lymph nodes $(n=9)$ underwent pelvic lymphadenectomy.

The median age of the 48 patients was 51 years (range, 31-70 years), 45 (93.75\%) of which had cervical squamous cell carcinoma and $3(6.25 \%)$ of which had adenocarcinoma. A medical history, physical examination, gynecologic pelvic examination, complete blood cell count, blood chemistry profile, chest X-ray or computed tomography (CT) scan, abdominal CT scan, and pelvic magnetic resonance imaging (MRI)/CT scan were obtained for all patients. In addition, 7 patients received positron emission tomography (PET). The patients were staged according to the staging system of the International Federation of Gynecology and Obstetrics (14), as follows: 3 patients $(6.3 \%)$ had stage IB disease, 1 patient $(2.1 \%)$ had stage IIA disease, 25 patients $(52.1 \%)$ had stage IIB disease, 3 patients $(6.3 \%)$ had stage IIIA disease and 16 patients (33.3\%) had stage IIIB disease (Table I).

Radiotherapy technique. All patients underwent CT-based planning with custom immobilization. The clinical target volume (CTV) included $\mathrm{CTV}_{1}$ and $\mathrm{CTV}_{2}$. CTV 1 consisted of $\mathrm{CTV}_{\text {cervix }}$ (including the gross tumor and cervix), $\mathrm{CTV}_{\text {uterus }}$ (including total uterus only), $\mathrm{CTV}_{\text {parametria }}$ [including the parametria; the entire mesorectum was added to the parametrial volume and superior half of the vagina (for patients with stage IIIA disease, the entire vagina was included] and $\mathrm{CTV}_{\text {node }}$ [including regional lymph nodes (common, internal, external iliac, obturator and presacral lymph nodes], according to the consensus guidelines $(15,16)$. Different margins were added to form the planning target volume (PTV). A $10-15 \mathrm{~mm}$ planning margin was applied around the cervix and gross tumor, a $15-20 \mathrm{~mm}$ margin around the uterus, and a $7 \mathrm{~mm}$ margin around the remainder of the $\mathrm{CTV}_{1}$ to form $\mathrm{PTV}_{1}$. By contrast, $\mathrm{CTV}_{2}$ included the para-aortic lymph nodes region, the lower border (bifurcation of abdominal aorta) and the upper border ( $7 \mathrm{~mm}$ below the T12/L1 interspace). A $7 \mathrm{~mm}$ planning margin was applied around $\mathrm{CTV}_{2}$ to define $\mathrm{PTV}_{2}$. The gross tumor volume of nodes (GTVnd) included enlarged lymph nodes, and a $5 \mathrm{~mm}$ margin was used around the GTVnd to produce the pGTVnd. The prescribed dose of $\mathrm{PTV}_{1}$ ranged between 45 and 50.4 Gy in 1.8-2.0 Gy daily fractions lasting 7-8 min, and of $\mathrm{PTV}_{2}$ ranged between 39.6 and $50 \mathrm{~Gy}$ in 1.8-2.0 Gy daily fractions lasting 7-8 min. Fifteen patients received the same prescribed dose for $\mathrm{PTV}_{1}$ and $\mathrm{PTV}_{2}$, while the others received the different prescribed dose for $\mathrm{PTV}_{1}$ and $\mathrm{PTV}_{2}$. pGTVnd was treated with a total dose of 55-60 Gy in 2.0-2.4 Gy daily fractions using simultaneous integrated boost-IMRT (Fig. 1). The target planning constraints were as follows: i) $>99 \%$ of the PTV received $>90 \%$ of the prescribed dose and $>97 \%$ PTV received $>97 \%$ of the prescribed dose; and ii) $<1 \%$ of the PTV received $>115 \%$ of the prescribed dose. The maximum dose applied to all tissues was $<115-117 \%$ of the prescription dose. The normal tissue planning constraints were as follows: i) Rectum [volume receiving $>50$ Gy (V50), <30\%; maximum dose, $<52 \mathrm{~Gy}$; ii) small bowel [volume receiving $>45$ Gy (V45), $<250 \mathrm{~cm}^{3}$ ]; iii) bladder [volume receiving $>50$ Gy (V50), <30\%; maximum dose, <52 Gy]; iv) bone marrow [volume receiving $>20$ Gy $(\mathrm{V} 20),<75 \%$; volume receiving $>10$ Gy (V10), <95\% (35/48 patients were planned prior to the introduction of bone marrow sparing)]; v) kidney [volume receiving >20 Gy (V20), <20\%; mean dose, <18 Gy] vi) liver [volume receiving $>30$ Gy $(\mathrm{V} 30),<40 \%$ ]; and vii) spinal cord (maximum dose, $<45$ Gy). Radiotherapy was suspended when the peripheral neutrophil count was $<500 / \mathrm{mm}^{3}$ or the peripheral platelet count was $<50,000 / \mathrm{mm}^{3}$ until the patient recovered. Patients were treated with a Varian Trilogy System Linear Accelerator (Varian Medical Systems, Palo Alto, CA, USA) at Anhui Tumor Hospital while Elekta Synergy (Elekta, Crawley, UK) was used at Anhui Provincial Hospital. All IMRT treatments were planned with Pinnacle ${ }^{3}$ v 9.10 (Philips Medical Systems, Fitchburg, WI, USA), and delivered using the step and shoot mode.

Following EF-IMRT, all patients underwent high dose-rate (HDR) intracavitary brachytherapy using iridium-192. Five fractions of 5.5-6.0 Gy each were delivered to point A (defined as $2 \mathrm{~cm}$ lateral to the central canal of the uterus and $2 \mathrm{~cm}$ up from the mucous membrane of the lateral fornix in the axis of the uterus) once or twice weekly, with no EF-IMRT treatment administered on the day of the HDR intracavitary treatment.

Chemotherapy. Nedaplatin, developed by Jiangsu Aosaikang Pharmaceutical Co., Ltd. (Nanjing, China), was dissolved in $500 \mathrm{ml}$ saline and infused intravenously. The first cycle was administered on the first day of EF-IMRT. All patients received weekly nedaplatin at a median dose of $30 \mathrm{mg} / \mathrm{m}^{2}$ (range, $25-40 \mathrm{mg} / \mathrm{m}^{2}$ ) once a week for 5 weeks (a total dose 
Table I. Patient characteristics $(n=48)$.

\begin{tabular}{|c|c|}
\hline Characteristic & Patients, n $(\%$ \\
\hline \multicolumn{2}{|l|}{ Age, years } \\
\hline Median & 51 \\
\hline Range & $31-70$ \\
\hline \multicolumn{2}{|l|}{ Histopathology } \\
\hline Squamous & $45(93.8)$ \\
\hline Adenocarcinoma & $3(6.3)$ \\
\hline \multicolumn{2}{|l|}{ FIGO stage } \\
\hline IB2 & $3(6.3)$ \\
\hline IIA & $1(2.1)$ \\
\hline IIB & $25(52.1)$ \\
\hline IIIA & $3(6.3)$ \\
\hline IIIB & $16(33.3)$ \\
\hline \multicolumn{2}{|l|}{ Lymph node involvement } \\
\hline Para-arotic nodes positive only & $12(25.0)$ \\
\hline Pelvic nodes positive only & $17(35.4)$ \\
\hline Para-aortic and pelvic nodes positive & $19(39.6)$ \\
\hline \multicolumn{2}{|c|}{ Treatment response according to FIGO stage } \\
\hline \multicolumn{2}{|l|}{ IB2 } \\
\hline CR & $3(6.3)$ \\
\hline PR & $0(0.0)$ \\
\hline \multicolumn{2}{|l|}{ IIA } \\
\hline CR & $1(2.1)$ \\
\hline PR & $0(0.0)$ \\
\hline \multicolumn{2}{|l|}{ IIB } \\
\hline CR & $25(52.1)$ \\
\hline PR & $0(0.0)$ \\
\hline \multicolumn{2}{|l|}{ IIIA } \\
\hline CR & $2(4.2)$ \\
\hline PR & $1(2.1)$ \\
\hline \multicolumn{2}{|l|}{ IIIB } \\
\hline CR & $15(31.3)$ \\
\hline PR & $1(2.1)$ \\
\hline
\end{tabular}

FIGO, Federation of Gynecology and Obstetrics; CR, complete response; PR, partial response.

of $150 \mathrm{mg} / \mathrm{m}^{2}$ every 5 weeks) during the course of EF-IMRT. Anti-emetics, such as 5-hydroxytryptamine-3 receptor antagonist, were administered routinely prior to nedaplatin infusion. However, nedaplatin infusion was delayed if the peripheral neutrophil count was $<1,000 / \mathrm{mm}^{3}$ or the peripheral platelet count was $<75,000 / \mathrm{mm}^{3}$. The optimal number of chemotherapy cycles administered was 5 .

Evaluation of treatment efficacy and follow-up of patients. During treatment, all the patients underwent weekly physical examinations, complete blood counts, and liver and renal function tests prior to the concurrent chemotherapy. Patient response
Table II. Treatment responses and failures.

Treatment responses and failures

Patients, $\mathrm{n}$

Treatment response

CR

46

PR

2

Treatment failure

$\mathrm{PR}$ and metastasis to the lung $\quad 1$

$\mathrm{CR}$ and recurrence and metastasis to the lung 2

$\mathrm{CR}$ and distant metastasis

Liver only 1

Lung only 1

Inguinal lymph nodes only 1

Supraclavicular lymph nodes $\quad 1$

Inguinal lymph nodes and lung 1

Inguinal lymph nodes and bone 1

Mediastinal lymph nodes only 1

Mediastinal and supraclavicular lymph nodes 2

$\mathrm{CR}$, complete response; $\mathrm{PR}$, partial response.

was evaluated for 3 months after completion of the treatment. Complete response (CR) was defined clinically (gynecological pelvic examination and imaging) as the disappearance of all gross lesions. Partial response (PR) was defined as a $>50 \%$ reduction of the tumor. Stable disease (SD) was defined as the presence of the tumor with $<50 \%$ reduction of the tumor size. Progressive disease (PD) was defined as a $>25 \%$ increase in the size of the local tumor or the appearance of any new lesion.

The treatment responses of all the patients were assessed by a radiation oncologist and/or a gynecological oncologist at 4 weeks and 3 months after the completion of treatment, and then followed up every 3 months during the first 2 years, and every 6 months thereafter. Follow-up evaluation included physical examination, gynecological pelvic examination, abdominal ultrasound or CT, pelvic ultrasound or CT and/or MRI, blood counts, and chemistry profiles. These patients were followed up regularly and the most recent follow-up was performed in August 2014.

Evaluation of treatment toxicities. Acute toxicity was measured from initiation of EF-IMRT to 90 days after the completion of treatment, while late toxicity was measured from $>90$ days after the completion of the treatment. Acute toxicity was graded according to the National Cancer Institute Common Terminology Criteria for Adverse Events version 3.0 (17) and late toxicity was graded according to the Radiation Therapy Oncology Group (RTOG) (18) late radiation morbidity scoring criteria.

Statistical analysis. Disease-free survival (DFS) and OS rate were analyzed using Kaplan-Meier curves, and treatment outcome was estimated by using Kaplan-Meier curves, in addition to the log-rank test for univariate analysis and the Cox proportional hazards model for multivariate analysis. SPSS 
Table III. Treatment toxicities.

\begin{tabular}{|c|c|c|c|c|c|}
\hline \multirow[b]{2}{*}{ Toxicity } & \multicolumn{5}{|c|}{ Cases, n (\%) } \\
\hline & Grade 0 & Grade 1 & Grade 2 & Grade 3 & Grade 4 \\
\hline \multicolumn{6}{|l|}{ Acute } \\
\hline Diarrhea & $22(45.8)$ & $11(22.9)$ & $13(27.1)$ & $2(4.2)$ & $0(0.0)$ \\
\hline Vomiting & $32(66.7)$ & $9(18.8)$ & 7 (14.6) & $0(0.0)$ & $0(0.0)$ \\
\hline Genitourinary & 45 (93.8) & $2(4.2)$ & $1(2.1)$ & $0(0.0)$ & $0(0.0)$ \\
\hline \multicolumn{6}{|l|}{ Hematological } \\
\hline Anemia & 19 (39.6) & $16(33.3)$ & $9(18.8)$ & $4(8.3)$ & $0(0.0)$ \\
\hline Leukopenia & $1(2.1)$ & $9(18.8)$ & $17(35.4)$ & $18(37.5)$ & $3(6.3)$ \\
\hline Neutropenia & $1(2.1)$ & $9(18.8)$ & $18(37.5)$ & $19(39.0)$ & $1(2.1)$ \\
\hline Thrombcytopenia & $22(45.8)$ & $15(31.3)$ & 7 (14.6) & $3(6.3)$ & $1(2.1)$ \\
\hline \multicolumn{6}{|l|}{ Late } \\
\hline Gastrointestinal & $33(68.8)$ & $3(6.3)$ & $10(20.8)$ & $2(4.2)$ & $0(0.0)$ \\
\hline Genitourinary & $43(89.6)$ & $2(4.2)$ & $3(6.3)$ & $0(0.0)$ & $0(0.0)$ \\
\hline
\end{tabular}
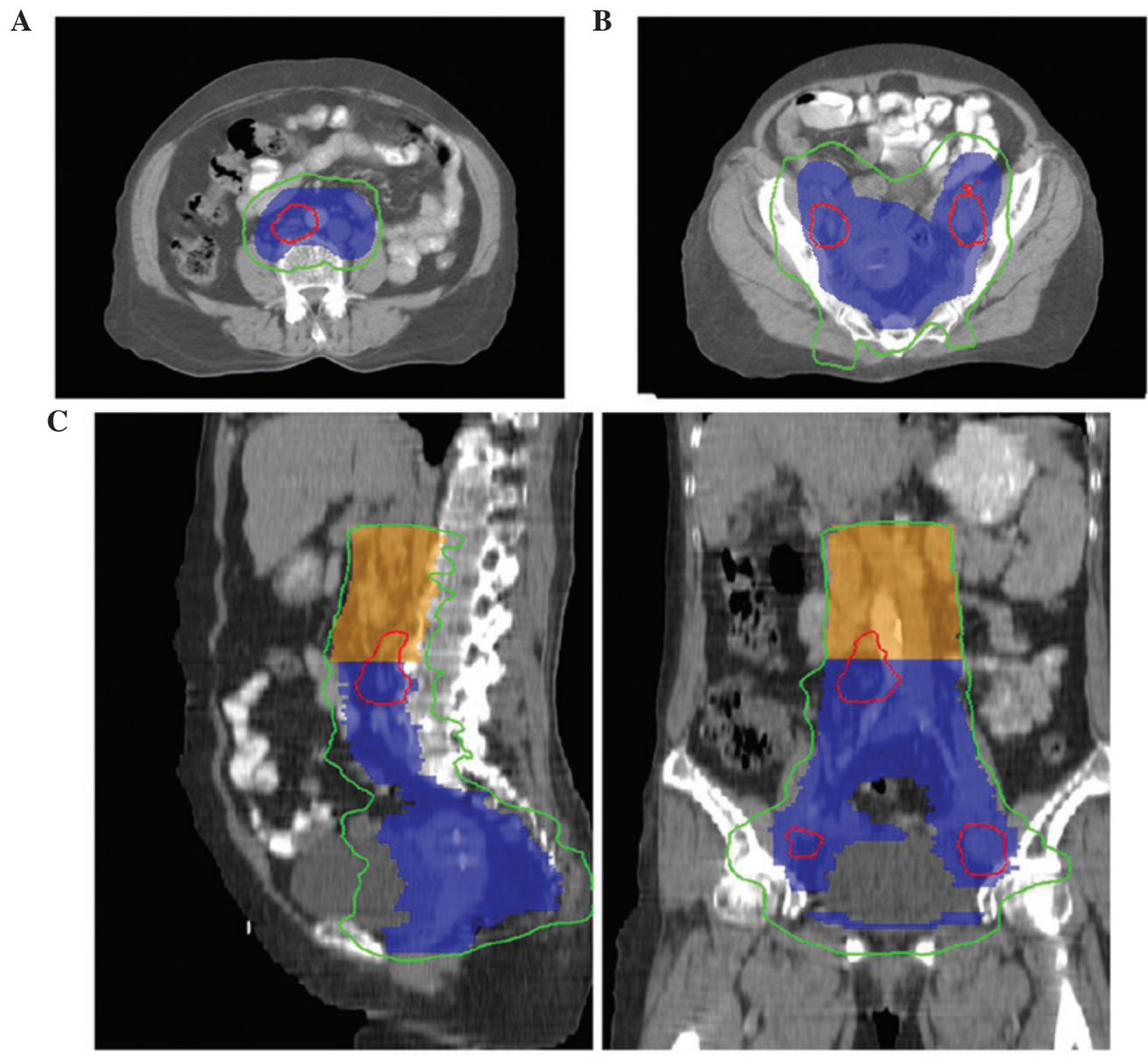

Figure 1. Illustration of radiation treatment doses from a single representative patient. Planning target volume (PTV), including PTV (blue) and PTV $_{2}($ orange), was covered by $45.0 \mathrm{~Gy}$ (green line). The PTV of involved lymph nodes was covered by $57.5 \mathrm{~Gy}$ (red line). (A) Transverse view of para-aortic lymph nodes; (B) transverse view of pelvic PTV; and (C) sagittal (left) and coronary (right) view of the isodose.

software (version 13.0; SPSS, Inc., Chicago, IL, USA) was used to perform the statistical analyses. Data are presented as the mean \pm standard deviation, and $\mathrm{P}<0.05$ was considered to indicate a statistically significant difference.

\section{Results}

Treatment response. Patient response was evaluated for 3 months after the completion of treatment. It was found that 
A

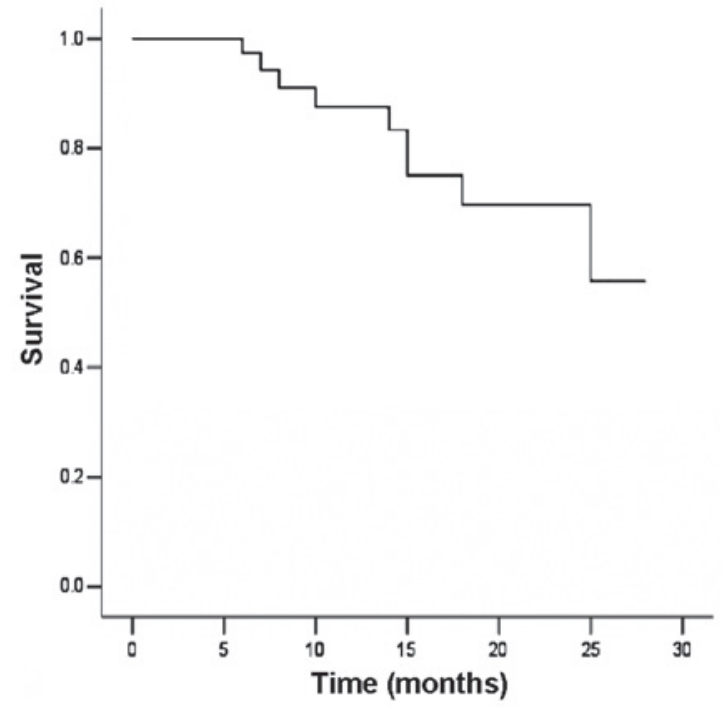

B

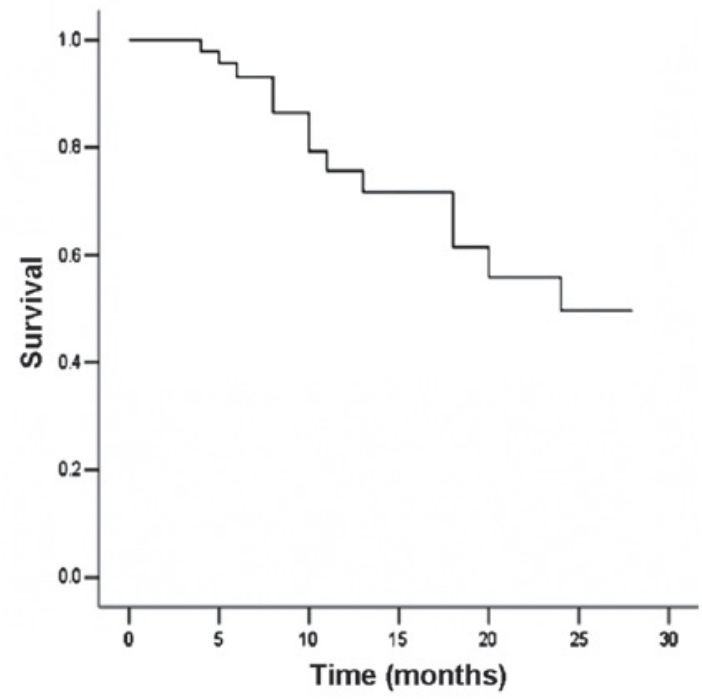

Figure 2. Kaplan-Meier curve analysis of (A) overall and (B) disease-free survival of 48 patients following extended field intensity-modulated radiotherapy plus concurrent chemotherapy.

46 patients had $\mathrm{CR}$ and 2 had $\mathrm{PR}$, with a response rate of $100 \%$ (Tables I and II). After 10 and 11 months, 2 patients with CR exhibited relapsed disease inside the radiation field and lung metastasis, while 9 patients with CR developed distant metastasis only. The most common site of distant metastasis was the lung (5 to the lung, 3 to the mediastinal lymph nodes, 3 to the inguinal lymph nodes, 3 to the supraclavicular lymph nodes, 1 to the liver and 1 to the lumbar vertebrae bone) (Table II).

OS and DFS. At the most recent follow-up in August 2014, $9 / 48$ patients $(18.8 \%)$ had succumbed to disease, between 6 and 25 months after treatment. Of these 9 patients, 2 had exhibited PR (one sucumbed to suicide at 8 months after therapy, the other succumbed to local tumor progression and distant metastasis). Among the remaining 7 patients, 1 patient succumbed due to local tumor relapse and distant metastasis, and 6 succumbed to distant metastasis. The median follow-up time was 12 months (range, 4-28 months). The 12-month OS and DFS were 87.5 and $75.8 \%$, respectively, and the 24-month OS and DFS were 69.7 and 49.7\%, respectively (Fig. 2).

Treatment toxicities. None of the 48 patients succumbed within 1 month after treatment, although 20/48 patients (41.7\%) had grade 3 or higher neutropenia, 4 patients $(8.3 \%)$ had grade 3 or higher thrombocytopenia, and 2 patients (4.2\%) had grade 3 diarrhea. No patients developed grade 3 or higher vomiting (Table III).

Specifically, 44/48 patients $(91.7 \%)$ were able to complete 5 weeks of concurrent nedaplatin chemotherapy, and only 4 patients $(8.3 \%)$ received $3 / 4$ cycles of chemotherapy due to grade 4 hematologic toxicity. A platelet transfusion was required by 1 patient, however, all patients completed radiotherapy. The median treatment duration from EF-IMRT to the last day of brachytherapy was 52 days (range, 48-61 days) and $45 / 48$ patients $(93.75 \%)$ completed radiotherapy in 8 weeks. Furthermore, 2 patients $(4.2 \%)$ developed grade 3 late GI toxicity, 1 patient experienced a sigmoid stricture that required colostomy, and 1 patient a experienced small intestine obstruction that required surgical management.

\section{Discussion}

In the present study, patients with cervical cancer were treated with EF-IMRT plus concurrent nedaplatin therapy for $\leq 8$ weeks. All patients responded to this treatment regimen, although 24/48 (45\%) patients experienced grade 3 or 4 neutropenia and thrombocytopenia. However, this treatment regimen featured a low rate of high-grade GI toxicity. The 12-month OS and DFS were 87.5 and $75.8 \%$, respectively, and the 24-month OS and DFS were 69.7 and 49.7\%, respectively. Thus, the present study indicates that EF-IMRT plus concurrent nedaplatin treatment in cervical patients is a safe and highly effective treatment strategy.

EFRT causes known side-effects on the bone marrow and small bowel, and the addition of concurrent chemotherapy to EFRT only exacerbates its toxicity. Previously, a prospective phase II cooperative group clinical trial, in which para-aortic lymph nodes were treated with 4,500 cGy, reported grade 3-4 acute GI toxicity in $18.6 \%$ of patients. Furthermore, a late morbidity actuarial risk of $14 \%$ at 4 years primarily involved the rectum in cervical cancer patients treated with concurrent chemotherapy and EFRT (7). However, the use of the IMRT significantly reduced doses to the OAR, and decreased GI and hematological toxicities (12). Consensus guidelines for delineation of the CTV have been published $(15,16)$. The theoretical drawbacks of IMRT treatment include dose inhomogeneity within target volumes, longer treatment times than conventional radiotherapy and the uncertainty of organ motion. However, previous studies demonstrated the advantages of IMRT over EFRT delivered by three-dimensional conformal radiotherapy for extended field treatments (19-21). For example, Portelance et al (19) performed a dosimetric analysis to determine the feasibility of pelvic and para-aortic IMRT in 10 patients who underwent CT simulation from $\mathrm{T} 2$ to the ischial tuberosities with conventional 2- and 4-field plans versus 4-, 
7- and 9-fields IMRT plans. IMRT resulted in a statistically significant reduction $(\mathrm{P}<0.05)$ in the volume of small bowel irradiation, demonstrating 11,15 and $13.6 \%$ reduction in the 4-, 7- and 9-field IMRT, respectively. By contrast, 35 and 34\% reduction occurred in the conventional 2- and 4-field plans, respectively. The rectal dose was significantly lower with IMRT $(\mathrm{P}<0.001)(19)$. Furthermore, Gerszten et al demonstrated a significant reduction in critical organ irradiation with EF-IMRT, and proposed that the treatment may reduce both acute and late treatment-associated side-effects (20). Salama et al reported the preliminary outcome and toxicity of EF-IMRT for gynecological malignancies, in which 13 women with gynecologic malignancies tolerated EF-IMRT and only 2 patients experienced grade 3 or higher acute toxicity (21). By contrast, Jensen et al demonstrated high toxicity rates in patients with cervical cancer that underwent EF-IMRT and concurrent cisplatin treatment; acute grade 3 or higher GI toxicity was $19.0 \%$ and acute grade 3 or higher hematological toxicity was $57.1 \%$ (22). In the current study, 20/48 patients $(41.7 \%)$ exhibited grade 3 or higher neutropenia, 4 patients $(8.4 \%)$ had grade 3 or higher thrombocytopenia and 2 patients (4.2\%) developed grade 3 diarrhea. This further indicates that the hematological toxicity was significant, however, the majority of patients tolerated the side effects, with $>90 \%$ of patients able to complete the treatment regime.

Nedaplatin is an analog of cisplatin that exhibits less nephrotoxicity, neurotoxicity and GI toxicity than cisplatin itself. A previous phase I study determined that the recommended weekly dose of nedaplatin was $30 \mathrm{mg} / \mathrm{m}^{2}$, administered for $>5$ cycles and $\leq 8$ cycles if possible. Weekly administration of nedaplatin may be more tolerable and less toxic than weekly administration of cisplatin (23). Furthermore, a phase II study confirmed that concurrent chemoradiotherapy using weekly nedaplatin $\left(30 \mathrm{mg} / \mathrm{m}^{2}\right)$ is safe and effective (24). No grade 3 vomiting was observed in the present study, and the total high-grade GI toxicity was $4.2 \%$, which is considerably lower than reported in a previous study by Jensen et al (22).

The impact of total length of radiation treatment is critical for determining OS rate. Walker et al reported that delays in treatment were predominantly a result of dehydration due to diarrhea, nausea and vomiting, which may be caused by cisplatin toxicity and increased small bowel radiation toxicity in the para-aortic field (25). With the integrated boost of involved nodes using EF-IMRT, the median treatment time from the first day of EF-IMRT to the last day of brachytherapy in the current study was 53 days (range, 48-61 days), which did not significantly prolong the length of radiation treatment compared with previous studies of chemoradiotherapy (7,25). Furthermore, regarding renal toxicity in patients treated with EFRT $(3,5)$, the current study did not induce any acute or late renal grade 3 or higher toxicity. Jensen et al reported 18-month cumulative incidences of late grade 3 or higher genitourinary and GI toxicity were 4.8 and $0 \%$, respectively, following EF-IMRT with concurrent cisplatin chemotherapy (22). RTOG 90-01 reported a late toxicity rate of $12 \%$ in patients treated with EFRT without concurrent chemotherapy (26). In prior studies of EFRT and concurrent chemotherapy, the reported late toxicity rates were $0-14 \%(7,27-30)$. The present study revealed that $2 / 48$ patients $(4.2 \%)$ developed grade 3 GI late toxicity. The current results are consistent with reports from these prior studies (7,27-31).

The delivery of radiation to grossly involved nodes has been limited due to its toxicity to adjacent organs and tissues (6). However, IMRT has been shown to permit delivery of $\leq 60$ Gy to para-aortic and/or pelvic nodes, with substantially reduced doses to the bowel, bladder and bone marrow $(17,32)$. In the current study, tumors metastasized to the para-aortic and/or pelvic nodes were treated with a total dose of 55-60 Gy in 2.0-2.4 Gy daily fractions using simultaneous integrated boost-IMRT. Following this treatment, no recurrence was observed in the para-aortic regions, and no increased acute or late GI toxicity was observed.

The present study demonstrated that concurrent treatment with nedaplatin and definitive EF-IMRT was effective in cervical cancer patients, with a low incidence of high-grade acute gastrointestinal toxicity. The results also revealed that distant metastasis was the major cause of mortality; thus, novel treatment regimens, such as multidrug concurrent chemo/radiotherapy or immunotherapy with or without radiotherapy, require investigation. The current study was not a randomized controlled trial and was limited by the small number of patients. Thus, in order to confirm the safety and efficacy of this treatment regime on patients with cervical cancer, further studies are warranted using a larger sample size and a randomized controlled trial.

\section{Acknowledgements}

We thank Medjaden Bioscience Limited (Hong Kong, China) for editing and proofreading this manuscript.

\section{References}

1. Jemal A, Bray F, Center MM, Ferlay J, Ward E and Forman D: Global cancer statistics. CA Cancer J Clin 61: 69-90, 2011.

2. Kumar V, Mitchell RS, Abbas AK and Fausto N (eds): The female genital system and breast. In: Robbins Basic Pathology. 8th edition. Saunders Elsevier, Philadelphia, PA, pp345-355, 2007.

3. Canavan TP and Doshi NR: Cervical cancer. Am Fam Physician 61: 1369-1376, 2000.

4. Waggoner SE: Cervical cancer. Lancet 361: 2217-2225, 2003.

5. Berman ML, Keys H, Creasman W, DiSaia P, Bundy B and Blessing J: Survival and patterns of recurrence in cervical cancer metastatic to periaortic lymph nodes (a Gynecologic Oncology Group study). Gynecol Oncol 19: 8-16, 1984.

6. Piver MS, Barlow JJ and Krishnamsetty R: Five-year survival (with no evidence of disease) in patients with biopsy-confirmed aortic node metastasis from cervical carcinoma. Am J Obstet Gynecol 139: 575-578, 1981.

7. Varia MA, Bundy BN, Deppe G, Mannel R, Averette HE, Rose PG and Connelly P: Cervical carcinoma metastatic to para-aortic nodes: Extended field radiation therapy with concomitant 5-fluorouracil and cisplatin chemotherapy: A Gynecologic Oncology Group study. Int J Radiat Oncol Biol Phys 42: 1015-1023, 1998.

8. Kim YS, Kim JH, Ahn SD, Lee SW, Shin SS, Nam JH, Kim YT, Kim YM, Kim JH and Choi EK: High-dose extended-field irradiation and high-dose-rate brachytherapy with concurrent chemotherapy for cervical cancer with positive para-aortic lymph nodes. Int J Radiat Oncol Biol Phys 74: 1522-1528, 2009.

9. Grigsby PW, Heydon K, Mutch DG, Kim RY and Eifel P: Long-term follow-up of RTOG 92-10: Cervical cancer with positive para-aortic lymph nodes. Int J Radiat Oncol Biol Phys 51: 982-987, 2001.

10. Small W Jr, Winter K, Levenback C, Iyer R, Gaffney D, Asbell S, Erickson B, Jhingran A and Greven K: Extended-field irradiation and intracavitary brachytherapy combined with cisplatin chemotherapy for cervical cancer with positive para-aortic or high common iliac lymph nodes: Results of ARM 1 of RTOG 0116. Int J Radiat Oncol Biol Phys 68: 1081-1087, 2007. 
11. Nugent EK, Case AS, Hoff JT, Zighelboim I, DeWitt LL, Trinkhaus K, Mutch DG, Thaker PH, Massad LS and Rader JS: Chemoradiation in locally advanced cervical carcinoma: An analysis of cisplatin dosing and other clinical prognostic factors. Gynecol Oncol 116: 438-441, 2010.

12. Carballo N, González-Cortijo L, González-Martin A, Rojo A and Chiva L: Indications for adjuvant radiotherapy treatment after surgery and novel modalities for treatment. Gynecol Oncol 110 (Suppl 2): S41-S44, 2008.

13. Desoize B and Madoulet C: Particular aspects of platinum compounds used at present in cancer treatment. Crit Rev Oncol Hematol 42: 317-325, 2002.

14. Pecorelli S, Zigliani L and Odicino F: Revised FIGO staging for carcinoma of the cervix. Int J Gynaecol Obstet 105: 107-108, 2009.

15. Lim K, Small W Jr, Portelance L, Creutzberg C, Jürgenliemk-Schulz IM, Mundt A, Mell LK, Mayr N, Viswanathan A, Jhingran A, et al: Consensus guidelines for delineation of clinical target volume for intensity-modulated pelvic radiotherapy for the definitive treatment of cervix cancer. Int J Radiat Oncol Biol Phys 79: 348-355, 2011

16. Small W Jr, Mell LK, Anderson P, Creutzberg C, De Los Santos J, Gaffney D, Jhingran A, Portelance L, Schefter T, Iyer R, et al: Consensus guidelines for delineation of clinical target volume for intensity-modulated pelvic radiotherapy in postoperative treatment of endometrial and cervical cancer. Int J Radiat Oncol Biol Phys 71: 428-434, 2008.

17. Cancer Therapy Evaluation Program: Common Terminology Criteria for Adverse Events, Version 3.0. ctep.cancer.gov/protocoldevelopment/electronic_applications/docs/ctcaev3.pdf\#search. Accessed, December 20, 2008.

18. Cox JD, Stetz J and Pajak TF: Toxicity criteria of the Radiation Therapy Oncology Group (RTOG) and the European Organization for Research and Treatment of Cancer (EORTC). Int J Radiat Oncol Biol Phys 31: 1341-1346, 1995.

19. Portelance L, Chao KS, Grigsby PW, Bennet H and Low D Intensity-modulated radiation therapy (IMRT) reduces small bowel, rectum, and bladder doses in patients with cervical cancer receiving pelvic and para-aortic irradiation. Int $\mathrm{J}$ Radiat Oncol Biol Phys 51: 261-266, 2001.

20. Gerszten K, Colonello K, Heron DE, Lalonde RJ, Fitian ID, Comerci JT, Selvaraj RN and Varlotto JM: Feasibility of concurrent cisplatin and extended field radiation therapy (EFRT) using intensity-modulated radiotherapy (IMRT) for carcinoma of the cervix. Gynecol Oncol 102: 182-188, 2006.

21. Salama JK, Mundt AJ, Roeske J and Mehta N: Preliminary outcome and toxicity report of extended-field, intensity-modulated radiation therapy for gynecologic malignancies. Int $\mathrm{J}$ Radiat Oncol Biol Phys 65: 1170-1176, 2006.

22. Jensen LG, Hasselle MD, Rose BS, Nath SK, Hasan Y, Scanderbeg DJ, Yashar CM, Mundt AJ and Mell LK: Outcomes for patients with cervical cancer treated with extended-field intensity-modulated radiation therapy and concurrent cisplatin. Int J Gynecol Cancer 23: 119-125, 2013.
23. Kodama J, Takemoto M, Seki N, Nakamura K, Hongo A, Kanazawa S and Hiramatsu Y: Phase I study of weekly nedaplatin and concurrent pelvic radiotherapy as adjuvant therapy after radical surgery for cervical cancer. Int J Gynecol Cancer 18: 1037-1041, 2008.

24. Niibe Y, Tsunoda S, Jobo T, Imai M, Matsuo K, Matsunaga K, Unno N and Hayakawa K: Phase II study of radiation therapy combined with weekly nedaplatin in locally advanced uterine cervical carcinoma (LAUCC): Kitasato Gynecologic Radiation Oncology Group (KGROG 0501)-initial analysis. Eur J Gynaecol Oncol 29: 222-224, 2008

25. Walker JL, Morrison A, DiSilvestro P and von Gruenigen VE; Gynecologic Oncology Group: A phase I/II study of extended field radiation therapy with concomitant paclitaxel and cisplatin chemotherapy in patients with cervical carcinoma metastatic to the para-aortic lymph nodes: A Gynecologic Oncology Group study. Gynecol Oncol 112: 78-84, 2009.

26. Eifel PJ, Winter K, Morris M, Levenback C, Grigsby PW, Cooper J, Rotman M, Gershenson D and Mutch DG: Pelvic irradiation with concurrent chemotherapy versus pelvic and para-aortic irradiation for high-risk cervical cancer: An update of radiation therapy oncology group trial (RTOG) 90-01. J Clin Oncol 22: 872-880, 2004.

27. Sood BM, Gorla GR, Garg M, Anderson PS, Fields AL, Runowicz CD, Goldberg GL and Vikram B: Extended-field radiotherapy and high-dose-rate brachytherapy in carcinoma of the uterine cervix: Clinical experience with and without concomitant chemotherapy. Cancer 97: 1781-1788, 2003.

28. Ring KL, Young JL, Dunlap NE, Andersen WA and Schneider BF: Extended-field radiation therapy with whole pelvis radiotherapy and cisplatin chemosensitization in the treatment of IB2-IIIB cervical carcinoma: A retrospective review. Am J Obstet Gynecol 201: 109.e1-e6, 2009.

29. Uno T, Mitsuhashi A, Isobe K, Yamamoto S, Kawakami H, Ueno N, Usui H, Tate S, Kawata T and Ito H: Concurrent daily cisplatin and extended-field radiation therapy for carcinoma of the cervix. Int J Gynecol Cancer 18: 80-84, 2008.

30. Chung YL, Jian JJ, Cheng SH, Hsieh CI, Tan TD, Chang HJ, Hung CF, Horng CF, Soong T and Tsou MH: Extended-field radiotherapy and high-dose-rate brachytherapy with concurrent and adjuvant cisplatin-based chemotherapy for locally advanced cervical cancer: A phase I/II study. Gynecol Oncol 97: 126-135, 2005.

31. Malfetano JH, Keys H, Cunningham MJ, Gibbons S and Ambros R: Extended field radiation and cisplatin for stage IIB and IIIB cervical carcinoma. Gynecol Oncol 67: 203-207, 1997.

32. Ahmed RS, Kim RY, Duan J, Meleth S, De Los Santos JF and Fiveash JB: IMRT dose escalation for positive para-aortic lymph nodes in patients with locally advanced cervical cancer while reducing dose to bone marrow and other organs at risk. Int $\mathrm{J}$ Radiat Oncol Biol Phys 60: 505-512, 2004. 